Pacific

Journal of

Mathematics

\title{
ON MASLOV CLASS RIGIDITY \\ FOR COISOTROPIC SUBMANIFOLDS
}

VIKTOR L. GINZBURG

Volume $250 \quad$ No. 1

March 2011 


\title{
ON MASLOV CLASS RIGIDITY FOR COISOTROPIC SUBMANIFOLDS
}

\author{
VIKTOR L. GINZBURG
}

\begin{abstract}
We define the Maslov index of a loop tangent to the characteristic foliation of a coisotropic submanifold as the mean Conley-Zehnder index of a path in the group of linear symplectic transformations, incorporating the "rotation" of the tangent space of the leaf - this is the standard Lagrangian counterpart - and the holonomy of the characteristic foliation. We also show that, with this definition, the Maslov class rigidity extends to the class of the so-called stable coisotropic submanifolds including Lagrangian tori and stable hypersurfaces.
\end{abstract}

\section{Introduction and main results}

1.1. Introduction. As the title indicates, the main theme of the paper is the Maslov class rigidity for coisotropic submanifolds. To be more specific, we define the Maslov index of a loop tangent to the characteristic foliation in a coisotropic submanifold and show that a displaceable, stable coisotropic submanifold carries a loop with Maslov index in the range [1, $2 n+1-k]$, where $2 n$ is the dimension of the ambient manifold and $k$ is the codimension of the coisotropic submanifold.

The study of symplectic topology of coisotropic submanifolds can be traced back to [Moser 1978] followed by [Banyaga 1980; Ekeland and Hofer 1989; Hofer 1990] and by the work of Bolle [1996; 1998]. Recently, the field has entered a particularly active phase; see [Albers and Frauenfelder 2010; 2008; Dragnev 2008; Ginzburg 2007; Gürel 2010; $\geq 2011$; Kang 2009; Kerman 2008; Tonnelier 2010; Usher 2009; Ziltener 2010; 2009]. Most of these papers, with the exception of [Ziltener 2009], concern such questions as generalizations to coisotropic submanifolds of the Lagrangian intersection property or of the existence of closed characteristics on stable hypersurfaces. The present work, which can be thought of as a follow-up to [Ginzburg 2007], focuses mainly on the coisotropic version of the Maslov class rigidity, also considered in [Ziltener 2009].

The work is partially supported by the NSF and by the faculty research funds of the University of California, Santa Cruz.

MSC2000: primary 53D40; secondary 37J45, 53D12.

Keywords: coisotropic submanifolds, Maslov class, Hamiltonian Floer homology. 
The aspect of the Maslov class rigidity we are concerned with here is the fact that the Maslov class of a closed displaceable Lagrangian submanifold automatically satisfies certain restrictions. Namely, the minimal Maslov number of such a submanifold lies between 1 and $n+1$. This phenomenon was originally studied in [Polterovich 1991a; 1991b; Viterbo 1990] and there are two methods of proving results of this type. One of these methods uses the holomorphic curves technique (see [Audin et al. 1994; Polterovich 1991a; 1991b]) and at this moment it is not known how to directly apply it to coisotropic submanifolds due to the lack of Fredholm properties for the Cauchy-Riemann problem with coisotropic boundary conditions. The second approach, originating from [Viterbo 1990], relies on Hamiltonian Floer homology (or its equivalent) and in combination with certain estimates from [Bolle 1998] can be easily adapted to the coisotropic setting; see, for example, [Ginzburg 2007]. Here, we heavily draw from the modern interpretation of this method given in [Kerman 2009; Kerman and Şirikçi 2010].

The Maslov index of a loop tangent to the characteristic foliation is the mean Conley-Zehnder index of a certain path in $\operatorname{Sp}(2 n)$ associated with the loop and comprising the "rotation" of the tangent space of the leaf, as the standard Lagrangian counterpart, and the holonomy of the characteristic foliation. Hence, the index can be an arbitrary real number. This definition, which can also be found in [Ziltener 2009], where it is treated in great detail, is of independent interest. Then, the proof of the Maslov class rigidity for coisotropic submanifolds follows the path of [Kerman 2009; Kerman and Şirikçi 2010; Viterbo 1990]. The main new element of the proof is that we circumvent relating the Conley-Zehnder and Morse indices as in [Duistermaat 1976; Viterbo 1990]; instead we use the explicit expression for the geodesic flow of a metric, capitalizing on the fact that the submanifolds in question are stable and hence admit a leaf-wise flat metric.

1.2. Coisotropic Maslov index. Let $M$ be a coisotropic submanifold of a symplectic manifold $\left(W^{2 n}, \omega\right)$. Denote by $\mathscr{F}$ the characteristic foliation of $M$; see Section 2.1 for the definition. The normal bundle $T^{\perp} M$ to $M$ is canonically isomorphic to the (leaf-wise) cotangent bundle $T^{*} \mathscr{F}$ to $\mathscr{F}$ and the direct sum $T \mathscr{F} \oplus T^{\perp} M$ is a symplectic vector bundle over $M$. We have a symplectic vector bundle decomposition

$$
\left.T W\right|_{M}=\left(T \mathscr{F} \oplus T^{\perp} M\right) \oplus T^{\perp} \mathscr{F},
$$

where $T^{\perp} \mathscr{F}$ is the normal bundle to $\mathscr{F}$ in $M$. Note that $T^{\perp} \mathscr{F}$ carries a symplectic leaf-wise flat connection.

Consider a loop $\gamma: S^{1} \rightarrow M$ tangent to $\mathscr{F}$, contractible in $W$ and equipped with a capping $u: D^{2} \rightarrow W$. The capping $u$ gives rise to a symplectic trivialization $\zeta$, unique up to homotopy, of the pull-back bundle $\gamma^{*} T W$. Let us assume first that 
$T \mathscr{F}$ is orientable along $\gamma$ (i.e., the pull-back $\gamma^{*} T \mathscr{F}$ is orientable), and hence trivial, and fix a trivialization $\xi$ of this vector bundle. Then the pull-back $\gamma^{*}\left(T \mathscr{F} \oplus T^{\perp} M\right)$ receives a symplectic trivialization $\xi \oplus \xi^{*}$. This trivialization can be viewed as a family of symplectic maps $\Xi(t): T_{\gamma(0)} \mathscr{F} \oplus T_{\gamma(0)}^{\perp} M \rightarrow T_{\gamma(t)} \mathscr{F} \oplus T_{\gamma(t)}^{\perp} M$ parametrized by $t \in S^{1}$. Combining the family $\Xi(t)$ with the holonomy $\Gamma(t): T_{\gamma(0)}^{\perp} \mathscr{F}_{\mathcal{F}} \rightarrow T_{\gamma(t)}^{\perp} \mathscr{F}$ along $\gamma$, we obtain a family of symplectic maps $\Xi(t) \oplus \Gamma(t): T_{\gamma(0)} W \rightarrow T_{\gamma(t)} W$, which, using the trivialization $\zeta$, we can regard as a path $\Phi:[0,1] \rightarrow \operatorname{Sp}(2 n)$.

Definition 1.1. The coisotropic Maslov index $\mu(\gamma, u)$ of the capped loop $(\gamma, u)$ is the negative mean Conley-Zehnder index $-\Delta(\Phi) \in \mathbb{R}$. (We refer the reader to [Long 2002; Salamon and Zehnder 1992] for a detailed discussion of the mean index; here we use the notation and conventions from [Ginzburg and Gürel 2009]; see Section 2.2.) When $T \mathscr{F}$ is not orientable along $\gamma$, we set $\mu(\gamma, u):=\mu\left(\gamma^{2}, u^{2}\right) / 2$, where $\left(\gamma^{2}, u^{2}\right)$ stands for the double cover of $(\gamma, u)$.

The standard argument shows that the index $\mu(\gamma, u)$ is well defined, that is, independent of the choice of the trivializations $\xi$ and $\zeta$. It is also independent of the choice of splitting (1-1): the normal bundle $T^{\perp} \mathscr{F}$ is unambiguously defined only as the quotient $T W / T \mathscr{F}$ while the splitting requires a choice of the complement to $T \mathscr{F}$ in $T W$. To see that $\Delta(\Phi)$ is independent of this choice, we argue as follows; see the proof of [Ginzburg and Gürel 2009, Lemma 2.6]. Observe that the path $\tilde{\Phi}$ resulting from a different splitting is homotopic to the concatenation of the path $\Phi$ with a path $\Psi$ of the form $\Psi(t)=I+A(t)$, where $I$ is the identity map and $A(t): T^{\perp \mathscr{F}} \rightarrow\left(T \mathscr{F} \oplus T^{\perp} M\right)$. Thus, all eigenvalues of $\Psi(t)$ are equal to one and, as a consequence, $\Delta(\Psi)=0$. Hence, by the additivity and homotopy invariance of the mean index [Ginzburg and Gürel 2009; Long 2002; Salamon and Zehnder 1992], we have $\Delta(\tilde{\Phi})=\Delta(\Phi)$.

It is worth emphasizing that, in contrast with the ordinary Lagrangian Maslov index, the coisotropic Maslov index is not, in general, an integer and that this index is different from the one considered in [Oh 2003]. The negative sign in the definition of the coisotropic Maslov index is, of course, a matter of conventions: this is the price we have to pay to match the sign of the standard Maslov index for Lagrangian submanifolds (Example 1.2) while using the conventions from [Ginzburg and Gürel 2009]; see Section 2.2.

It is easy to see that the coisotropic Maslov index has the following properties:

- Homotopy invariance: $\mu(\gamma, u)$ is invariant, in the obvious sense, under a homotopy of $\gamma$ in a leaf of $\mathscr{F}$. In particular, $\mu(\gamma, u)=0$ when $u$ is homotopic (rel boundary) to a disc in the leaf of $\mathscr{F}$ containing $\gamma$.

- Recapping: $\mu(\gamma, u \# v)=\mu(\gamma, u)-2\left\langle c_{1}(T W), v\right\rangle$, where the capping $u \# v$ is obtained by attaching the sphere $v \in \pi_{2}(W)$ to $u$. In particular, $\mu(\gamma):=\mu(\gamma, u)$ is independent of $u$ when $\left.c_{1}(T W)\right|_{\pi_{2}(W)}=0$. 
- Homogeneity: $\mu\left(\gamma^{k}, u^{k}\right)=k \mu(\gamma, u)$, where $\left(\gamma^{k}, u^{k}\right)$ stands for the $k$-fold cover of $(\gamma, u)$. Moreover, when $\left.c_{1}(T W)\right|_{\pi_{2}(W)}=0$, the Maslov index gives rise to a homogeneous quasimorphism $\pi_{1}(F) \rightarrow \mathbb{R}$ for any leaf $F$ of $\mathscr{F}$.

Example 1.2. When $M$ is a Lagrangian submanifold of $W$, the foliation $\mathscr{F}$ has only one leaf, the manifold $M$ itself, and the coisotropic Maslov index coincides with the ordinary Maslov index. Indeed, in this case, Definition 1.1 turns into one of the definitions of this index.

Example 1.3. When $u$ is contained in $M$, the index $\mu(\gamma, u)$ is equal to the mean index of the holonomy along $\gamma$ with respect to a symplectic trivialization of $T^{\perp} \mathscr{F}_{F}$ associated with $u$. For instance, when $M$ is a regular level of a Hamiltonian and $\gamma$ is a periodic orbit (and again $u$ is contained in $M$ ), the Maslov index $\mu(\gamma, u)$ is equal to the mean index of $\gamma$ in $M$.

Example 1.4. When all leaves of $\mathscr{F}$ are closed and form a fibration, the path $\Phi$ is a loop and $\mu(\gamma, u)$ is equal to the Maslov index of this loop. (In particular, then $\mu(\gamma, u)$ is an integer.) In this setting, the coisotropic Maslov index is introduced and investigated by Ziltener [2009]. Furthermore, one can express the coisotropic Maslov index via the Lagrangian Maslov index in the graph of $\mathscr{F}$; see [Ziltener 2009; 2010] for details.

Now we are in a position to state the main result of the paper. A much more detailed discussion of the coisotropic Maslov index can be found in [Ziltener 2009].

1.3. Rigidity of the coisotropic Maslov index. Let $W$ be a symplectically aspherical manifold, which we assume to be either closed or geometrically bounded and wide (e.g., convex at infinity) in the sense of [Gürel 2008].

Theorem 1.5. Let $W^{2 n}$ be as above and let $M^{2 n-k} \subset W$ be a closed, stable, displaceable coisotropic submanifold. (See Section 2.1 for the definitions.) Then, for any $\delta>0$, there exists a loop $\eta$ tangent to $\mathscr{F}$ and contractible in $W$ and such that

$$
\begin{aligned}
& 1 \leq \mu(\eta) \leq 2 n+1-k, \\
& 0<\operatorname{Area}(\eta) \leq e(M)+\delta,
\end{aligned}
$$

where $\operatorname{Area}(\eta)$ is the symplectic area bounded by $\eta$ and $e(M)$ is the displacement energy of $M$.

Example 1.6. As in Example 1.2, assume that $M$ is a stable Lagrangian submanifold (and hence a torus). Then $k=n$ and the theorem reduces to a particular case of the standard Lagrangian Maslov class rigidity. This version of rigidity is established in [Viterbo 1990] for $W=\mathbb{R}^{2 n}$ and in [Kerman 2009; Kerman and Şirikçi 2010] for closed ambient manifolds; see also [Audin et al. 1994; Polterovich 1991a; 1991b] for generalizations. 
Example 1.7. Assume that $M$ is a stable, displaceable, simply connected hypersurface. Then, by (1-2) and Example 1.3, $M$ carries a closed characteristic $\eta$ with $1 \leq \Delta(\gamma) \leq 2 n$. This is apparently a new observation. However, if we replace the upper bound by $2 n+1$, the assertion becomes an easy consequence of the properties of the mean index and, for instance, the displacement or symplectic homology proof of the almost existence theorem; see, for example, [Floer et al. 1994; Ginzburg 2005; Gürel 2008; Hofer and Zehnder 1994] and references therein.

Remark 1.8. A word on the hypotheses of the theorem is due now. The assumption that $W$ be symplectically aspherical is imposed here only for the sake of simplicity and can be significantly relaxed along the lines of [Kerman 2008; Usher 2009]. Hypothetically, a combination of our argument with the reasoning from these works should lead to a generalization of the theorem to the case where we only require the subgroup $\left\langle\omega, \pi_{2}(M)\right\rangle \subset \mathbb{R}$ to be discrete as in [Usher 2009, Theorem 1.6] or, at least, where $W$ is monotone or negative monotone; see [Kerman 2008]. (In such a generalization, the geodesic $\eta$ is, of course, equipped with capping.)

The condition that $M$ is stable cannot be entirely omitted due to the counterexamples to the Hamiltonian Seifert conjecture showing that there exist hypersurfaces in $\mathbb{R}^{2 n}\left(C^{2}\right.$ when $\left.2 n=4\right)$ without closed characteristics; see [Ginzburg 1999; Ginzburg and Gürel 2003] and references therein. However, this condition can possibly be relaxed as in [Usher 2009, Section 7].

Finally note that the existence of a loop $\eta$ satisfying (1-3) is established in [Ginzburg 2007, Theorem 2.7], where the second inequality (with $\delta=0$ ) is proved under the additional hypothesis that $M$ has restricted contact type. Thus, even when only the area bounds are concerned, Theorem 1.5 is a generalization (up to the issue of $\delta$ ) of the results from [Ginzburg 2007], which became possible due to incorporating a technique from [Kerman 2009; Kerman and Şirikçi 2010] into the proof.

Remark 1.9. It is tempting to conjecture that the Maslov class of $M$ is still nonzero even when the stability assumption in Theorem 1.5 is dropped and all leaves of $\mathscr{F}_{F}$ may be contractible. However, it is not entirely clear how to define this Maslov class and what cohomology space this class should lie in. The situation contrasts sharply with a similar question for the Liouville class of $M$, which can always be defined, when $W$ is exact, as the class $\left[\left.\lambda\right|_{\mathscr{F}}\right]$ of a global primitive $\lambda$ of $\omega$ in the tangential de Rham cohomology $\mathrm{H}^{1}(\mathscr{F})$; see [Ginzburg 2007, Section 1.2].

\section{Preliminaries}

We start this section by recalling the relevant definitions and basic results concerning coisotropic submanifolds. In Section 2.2, we set our conventions and notation. 
2.1. Stable coisotropic submanifolds. Let, as above, $\left(W^{2 n}, \omega\right)$ be a symplectic manifold and let $M \subset W$ be a closed, coisotropic submanifold of codimension $k$. Set $\omega_{M}=\left.\omega\right|_{M}$. Then, as is well known, the distribution $\operatorname{ker} \omega_{M}$ has dimension $k$ and is integrable. Denote by $\mathscr{F}$ the characteristic foliation on $M$, that is, the $k$-dimensional foliation whose leaves are tangent to the distribution $\operatorname{ker} \omega_{M}$.

Definition 2.1. The coisotropic submanifold $M$ is said to be stable if there exist one-forms $\alpha_{1}, \ldots, \alpha_{k}$ on $M$ such that $\operatorname{ker} d \alpha_{i} \supset \operatorname{ker} \omega_{M}$ for all $i=1, \ldots, k$ and

$$
\alpha_{1} \wedge \cdots \wedge \alpha_{k} \wedge \omega_{M}^{n-k} \neq 0
$$

anywhere on $M$. We say that $M$ has contact type if the forms $\alpha_{i}$ can be taken to be primitives of $\omega_{M}$. Furthermore, $M$ has restricted contact type if the forms $\alpha_{i}$ extend to global primitives of $\omega$ on $W$.

Stable and contact type coisotropic submanifolds were introduced by Bolle [1996; 1998] and considered in a more general setting in [Ginzburg 2007] and also by Kerman [2008] and Usher [2009]. We refer the reader to [Ginzburg 2007] for a discussion of the requirements of Definition 2.1 and examples. Here we only note that although Definition 2.1 is natural, it is quite restrictive. For example, a stable Lagrangian submanifold is necessarily a torus and a stable coisotropic submanifold is automatically orientable.

Assume henceforth that $M$ is stable. Then the normal bundle $T^{\perp} M$ to $M$ in $W$ is trivial, since it is isomorphic to $T^{*} \mathscr{F}$ and the latter bundle is trivial due to (2-1). From now on, we fix the trivialization $T^{\perp} M=T^{*} \mathscr{F} \cong M \times \mathbb{R}^{k}$ given by the forms $\alpha_{i}$ and identify a small neighborhood of $M$ in $W$ with a neighborhood of $M$ in $T^{*} \mathscr{F}=M \times \mathbb{R}^{k}$. We will use the same symbols $\omega_{M}$ and $\alpha_{i}$ for differential forms on $M$ and for their pullbacks to $M \times \mathbb{R}^{k}$. (Thus we suppress the pullback notation $\pi^{*}$, where $\pi: M \times \mathbb{R}^{k} \rightarrow M$ is the natural projection, unless its presence is essential.) As a consequence of the Weinstein symplectic neighborhood theorem, we have:

Proposition 2.2 [Bolle 1996; 1998]. Let M be a closed, stable coisotropic submanifold of $\left(W^{2 n}, \omega\right)$ with $\operatorname{codim} M=k$. Then, for a sufficiently small $r>0$, there exists a neighborhood of $M$ in $W$, which is symplectomorphic to

$$
U_{r}=\left\{(q, p) \in M \times \mathbb{R}^{k}|| p \mid<r\right\},
$$

equipped with the symplectic form $\omega=\omega_{M}+\sum_{j=1}^{k} d\left(p_{j} \alpha_{j}\right)$. Here $\left(p_{1}, \ldots, p_{k}\right)$ are the coordinates on $\mathbb{R}^{k}$ and $|p|$ is the Euclidean norm of $p$.

Thus, a neighborhood of $M$ in $W$ is foliated by a family of coisotropic submanifolds $M_{p}=M \times\{p\}$ with $p \in B_{r}^{k}$, where $B_{r}^{k}$ is the ball of radius $r$ centered at the origin in $\mathbb{R}^{k}$. Moreover, a leaf of the characteristic foliation on $M_{p}$ projects onto a leaf of the characteristic foliation on $M$. 
Proposition 2.3 [Bolle 1996; 1998; Ginzburg 2007]. Let M be a stable coisotropic submanifold.

(i) The leaf-wise metric $\left(\alpha_{1}\right)^{2}+\cdots+\left(\alpha_{k}\right)^{2}$ on $\mathscr{F}$ is leaf-wise flat.

(ii) The Hamiltonian flow of $\rho=\left(p_{1}^{2}+\cdots+p_{k}^{2}\right) / 2=|p|^{2} / 2$ is the leaf-wise geodesic flow of this metric.

We conclude this section by pointing out that the metric $\rho$ extends to a true metric on $M$ such that the leaves of $\mathscr{F}$ are totally geodesic submanifolds and that the existence of such a metric is equivalent to the stability of $M$ when $M$ is a hypersurface; see [Sullivan 1978] and [Usher 2009, Section 7].

2.2. Conventions and notation. In this section we specify conventions and notation used throughout the paper.

2.2.1. Action functional and the Hamilton equation. Let $\left(W^{2 n}, \omega\right)$ be a symplectically aspherical manifold, that is, $\left.\omega\right|_{\pi_{2}(W)}=\left.c_{1}\right|_{\pi_{2}(W)}=0$. Denote by $\Lambda W$ the space of smooth contractible loops $\gamma: S^{1} \rightarrow W$ and consider a time-dependent Hamiltonian $H: S^{1} \times W \rightarrow \mathbb{R}$, where $S^{1}=\mathbb{R} / \mathbb{Z}$. Setting $H_{t}=H(t, \cdot)$ for $t \in S^{1}$, we define the action functional $\mathscr{A}_{H}: \Lambda W \rightarrow \mathbb{R}$ by

$$
\mathscr{A}_{H}(\gamma)=\mathscr{A}(\gamma)+\int_{S^{1}} H_{t}(\gamma(t)) d t
$$

where $\mathscr{A}(\gamma)=-\operatorname{Area}(\gamma)$ is the negative symplectic area bounded by $\gamma$. In other words,

$$
\mathscr{A}(\gamma)=-\int_{u} \omega
$$

where $u: D^{2} \rightarrow W$ is a capping of $\gamma$, that is, $\left.u\right|_{S^{1}}=\gamma$. The least action principle asserts that the critical points of $\mathscr{A}_{H}$ are exactly the contractible one-periodic orbits of the time-dependent Hamiltonian flow $\varphi_{H}^{t}$ of $H$, where the Hamiltonian vector field $X_{H}$ of $H$ is defined by the Hamilton equation $i_{X_{H}} \omega=-d H$.

2.2.2. Conley-Zehnder index. We consider a finite-dimensional symplectic vector space $V$ and denote by $\operatorname{Sp}(V)$ the group of linear symplectic transformations of $V$, setting $\operatorname{Sp}(2 n)=\operatorname{Sp}\left(\mathbb{R}^{2 n}\right)$ as usual. We let $\Delta(\Phi)$ stand for the mean index of a path $\Phi:[0, T] \rightarrow \mathrm{Sp}(V)$ and, when $\Phi$ is nondegenerate (i.e., $\Phi(T)$ has no eigenvalues equal to one), we denote by $\mu_{\mathrm{CZ}}(\Phi)$ the Conley-Zehnder index of $\Phi$. We refer the reader to [Long 2002; Salamon 1999; Salamon and Zehnder 1992] and also [Ginzburg and Gürel 2009] for the definitions and a detailed discussion of these notions. In this paper, we normalize these indices as in [Ginzburg and Gürel 2009]. This normalization is different from the ones in [Long 2002; Salamon 1999; Salamon and Zehnder 1992]. For instance, our $\mu_{\mathrm{CZ}}(\Phi)$ is the negative of the Conley-Zehnder index as defined in [Salamon 1999]. For the flow $\Phi(t)$ 
with $0 \leq t \leq 1$ generated by a nondegenerate quadratic Hamiltonian $H$ with small eigenvalues, we have $\mu_{\mathrm{CZ}}(\Phi)=-\operatorname{sgn}(H) / 2$, where $\operatorname{sgn}(H)$ is the signature of $H$ (the number of positive squares minus the number of negative squares). In particular, when $H$ is negative definite, we have $\mu_{\mathrm{CZ}}(\Phi)=n$ where $2 n=\operatorname{dim} V$ and $\Delta(\Phi)>0$. In other words, when $\mu_{\mathrm{CZ}}(\Phi)$ is interpreted as the intersection index of $\Phi$ with the discriminant $\Sigma \subset \operatorname{Sp}(V)$ formed by symplectic transformations with at least one eigenvalue equal to one, $\Sigma$ is co-oriented by the Hamiltonian vector field of a negative definite Hamiltonian.

Recall also from [Salamon and Zehnder 1992] that, regardless of conventions, we have

$$
\left|\Delta(\Phi)-\mu_{\mathrm{CZ}}(\Phi)\right|<n \text { and } \Delta(\Phi)=\lim _{k \rightarrow \infty} \frac{\mu_{\mathrm{CZ}}\left(\Phi^{k}\right)}{k},
$$

where in the inequality we require $\Phi(T)$ to be nondegenerate and, in the limit identity, we assume that $\Phi(T)^{k} \notin \Sigma$ for all $k$ and thus $\mu_{\mathrm{CZ}}\left(\Phi^{k}\right)$ is defined. Note that here we can replace $\Phi^{k}$ by the concatenation of the paths $\Phi, \Phi(T) \Phi$, etc., up to $\Phi(T)^{k-1} \Phi$.

Let now $x$ be a contractible periodic orbit of $H$ on $W^{2 n}$. Using a trivialization of $x^{*} T W$ arising from a capping of $x$, we can interpret the linearized flow $d \varphi_{H}^{t}$ along $x$ as a path $\Phi$ in $\operatorname{Sp}(2 n)$. The mean index $\Delta(x)$ of $x$ is by definition $\Delta(\Phi)$. When $x$ is nondegenerate, we also set $\mu_{\mathrm{CZ}}(x):=\mu_{\mathrm{CZ}}(\Phi)$. Since $\left.c_{1}(T W)\right|_{\pi_{2}(W)}=0$, these indices are well defined, that is, independent of the capping. When we need to emphasize the role of $H$, we write $\Delta_{H}(x)$ and $\mu_{\mathrm{CZ}}(x, H)$. By (2-2), we have

$$
\left|\Delta(x)-\mu_{\mathrm{CZ}}(x)\right|<n \text { and } \Delta(x)=\lim _{k \rightarrow \infty} \frac{\mu_{\mathrm{CZ}}\left(x^{k}\right)}{k} .
$$

As in (2-2), we require here $x$ to be nondegenerate for $\mu_{\mathrm{CZ}}(x)$ to be defined, and, in the limit identity, we assume that $x$ is strongly nondegenerate, that is, all iterated orbits $x^{k}$ are nondegenerate. Finally note that with our normalizations $\Delta(x)>0$ and $\mu_{\mathrm{CZ}}(x)=n$ when $x$ is a nondegenerate maximum (with small Hessian) of an autonomous Hamiltonian.

2.2.3. Floer homology. In the definition of Floer homology, we adopt literally the conventions and notation from [Ginzburg 2007]. All Hamiltonians considered in this paper are assumed to be compactly supported. The manifold $W$, in addition to being symplectically aspherical, is required to be either closed or geometrically bounded and wide in the sense of [Gürel 2008]. (See, e.g., [Audin et al. 1994; Cieliebak et al. 2004; Sikorav 1994] for the precise definition and a discussion of geometrically bounded manifolds.)

Examples of geometrically bounded manifolds include symplectic manifolds which are convex at infinity (e.g., $\mathbb{R}^{2 n}$ and cotangent bundles) as well as twisted 
cotangent bundles. Under the hypotheses that $W$ is symplectically aspherical and geometrically bounded, the compactness theorem for Floer's connecting trajectories holds (see [Sikorav 1994]) and the filtered $\mathbb{Z}$-graded Floer homology of a compactly supported Hamiltonian on $W$ is defined for action intervals not containing zero; see, for example, [Cieliebak et al. 2004; Ginzburg and Gürel 2004] and references therein. We use the wideness hypothesis in Section 3.2 when considering a version of the "pinned" action selector introduced in [Kerman 2009]. This requirement is not restrictive, for, to the best of the author's knowledge, no examples of geometrically bounded open manifolds that are not wide are known.

We use the notation $\mathrm{HF}_{*}^{(a, b)}(H)$ for the filtered Floer homology of $H$, graded by the Conley-Zehnder index. The end-points $a$ and $b$ are always assumed to be outside the action spectrum $\mathscr{S}(H)$ of $H$ and, if $W$ is open, we require that $0 \notin(a, b)$. When $W$ is closed, we have a canonical isomorphism

$$
\mathrm{HF}_{*}(H)=\mathrm{H}_{*+n}\left(W ; \mathbb{Z}_{2}\right),
$$

where as usual $\mathrm{HF}_{*}(H)=\mathrm{HF}_{*}^{(-\infty, \infty)}(H)$. When all periodic orbits of $H$ with action in $(a, b)$ are nondegenerate, we let $\mathrm{CF}_{*}^{(a, b)}(H)$ be the vector space generated over $\mathbb{Z}_{2}$ by such orbits, graded by the Conley-Zehnder index. The downward Floer differential

$$
\partial: \mathrm{CF}_{*}^{(a, b)}(H) \rightarrow \mathrm{CF}_{*-1}^{(a, b)}(H)
$$

is then defined in the standard way and $\operatorname{HF}_{*}^{(a, b)}(H)$ is the homology of the resulting Floer complex. The above nondegeneracy requirement is generic (as long as $0 \notin$ $(a, b)$ if $W$ is open) and, in general, we set

$$
\mathrm{HF}_{*}^{(a, b)}(H):=\mathrm{HF}_{*}^{(a, b)}(\tilde{H}),
$$

where $\tilde{H}$ is a small perturbation of $H$ having only nondegenerate orbits with action in $(a, b)$. Since $a$ and $b$ are outside $\mathscr{Y}(H)$, the homology $\mathrm{HF}_{*}^{(a, b)}(\tilde{H})$ is independent of $\tilde{H}$ as long as $\tilde{H}$ is sufficiently close to $H$. We refer the reader to [Cieliebak et al. 2004; Ginzburg 2007; Ginzburg and Gürel 2004] for the proofs and further details on the construction and properties of the Floer homology in this setting as well as for further references.

\section{Proof of the main theorem}

3.1. Maslov index for stable coisotropic submanifolds. Let $M$ be a stable coisotropic submanifold. In this section, we interpret the mean index $\Delta_{\rho}(x)$ of a periodic orbit $x$ of the leaf-wise geodesic flow on $M$ as, up to a sign, the coisotropic Maslov index of the projection $\gamma$ of $x$ to $M$. We also establish certain bounds, going beyond (2-3), on the Conley-Zehnder index of a small nondegenerate perturbation of $x$. Throughout this subsection, we will use the notation from Section 2.1. In particular, 
we fix a neighborhood $U=M \times B$, where $B=B_{r}$, of $M$ in $W$. Thus, let $x$ be a nontrivial, contractible in $W$ closed orbit of the Hamiltonian flow of $\rho$ and let $\gamma=\pi(x)$. Then $\gamma$ is also contractible in $W$.

Proposition 3.1. We have

$$
\mu(\gamma)=-\Delta_{\rho}(x) .
$$

Proof. It is convenient to first extend the decomposition (1-1) from $\left.T W\right|_{M}$ to $\left.T W\right|_{U}$ as follows. Recall from Section 2.1 that the submanifolds $M_{p}=M \times\{p\} \subset M \times B$, with $p \in B$, are coisotropic and that the characteristic foliation $\mathscr{F}_{p}$ of $M_{p}$ projects to $\mathscr{F}$ under $\pi$. Denote by $\tilde{F}$ the resulting foliation of $U$, obtained as the union of foliations $\mathscr{F}_{p}$. Let $T M$ be the horizontal tangent bundle in $M \times B$, that is, $(T M)_{(q, p)}=T_{(q, p)} M_{p}$ where $(q, p) \in U=M \times B$, and likewise let $T B$ denote the vertical bundle $\operatorname{ker} \pi_{*}$. Then the normal bundle $T^{\perp} \tilde{\mathscr{F}}_{\mathrm{F}}$ to $T \tilde{\mathscr{F}}_{\mathrm{F}}$ in $T M$ can be realized as the subbundle $E=\left(\cap_{i} \operatorname{ker} \pi^{*} \alpha_{i}\right) \cap T M$. We have the symplectic decomposition

$$
T W=(T \tilde{\tilde{F}} \oplus T B) \oplus E,
$$

which turns into (1-1) once restricted to $M$.

The linearized projection $\pi_{*}$ gives rise to an isomorphism between the fibers $\left(T \tilde{\mathscr{F}}_{(q, p)}\right.$ and $T_{q} \mathscr{F}_{F}$, and $E_{(q, p)}$ and $T_{q}^{\perp} \mathscr{F}_{\text {. Furthermore, }}(T B)_{(q, p)}$ is naturally isomorphic to $T_{0} B=T_{q}^{\perp} M$. Thus, we have a (symplectic) linear isomorphism between the decomposition (3-2) along $x$ and (1-1) along $\gamma$. In particular, we obtain an isomorphism between the bundles $x^{*} T W$ and $\gamma^{*} T W$ giving rise to a one-to-one correspondence between trivializations of $T W$ along $x$ and along $\gamma$. In what follows, we fix a trivialization arising from a capping of $x$.

Now recall that the flow of $\rho$ on $U$ can be identified with the geodesic flow of the leaf-wise metric $\rho$ on $M$. Thus, we need to prove that the mean index of the linearized geodesic flow $G(t)$ along $x$ is equal to $\Delta(\Phi)$. The geodesic flow preserves the terms $T \tilde{\mathscr{F}} \oplus T B$ and $E$ in the decomposition (3-2). Indeed, the fact that the first term is conserved is clear: the geodesic flow is tangent to the leaves. To show that the second term is conserved, it suffices to recall that, as mentioned above, the flow is tangent to the manifolds $M_{p}$ due to conservation of momenta and that the restrictions $\left.\pi^{*} \alpha_{j}\right|_{M_{p}}$ are conserved since $L_{X_{\rho}} \pi^{*} \alpha_{j}=d p_{j}$.

Next let us show that

$$
\left.G\right|_{E}=\Gamma,
$$

where we identified $x^{*} E$ and $\gamma^{*} T^{\perp \mathscr{F}}$. To this end, let us recall the definition of the holonomy $\Gamma$. Consider an element $[v]$ in $T_{\gamma(0)}^{\perp} \mathscr{F}_{\mathscr{F}}=T_{\gamma(0)} M / T_{\gamma(0)} \mathscr{F}_{\mathscr{F}}$ represented by a vector $v \in T_{\gamma(0)} M$. (Here and below, it is more convenient to think of $E$ and 
$T^{\perp} \mathscr{F}$ as quotient bundles rather than sub-bundles.) Let $\eta:[0, \delta) \rightarrow M$ be a smooth map with $\eta(0)=\gamma(0)$ and $\eta^{\prime}(0)=v$. Let now $\gamma$ be parametrized by, say, [0,T] and let $\sigma:[0, T] \times[0, \delta) \rightarrow M$ be a map whose restriction to $[0, T] \times 0$ is $\gamma$, to $0 \times[0, \delta)$ is $\eta$ and such that $\left.\sigma\right|_{[0, T] \times s}$, for all $s \in[0, \delta)$, lies in a leaf of $\mathscr{F}$. The class $[(\partial \sigma / \partial s)(t, 0)] \in T_{\gamma(t)}^{\perp} \mathscr{F}$ is independent of the choice of $\sigma$ and is the image $\Gamma(t)[v]$. Let now $w(s) \in T_{\eta(s)} \mathscr{F}$ be a smooth family of vectors tangent to $\mathscr{F}$ and such that $w(0)=\dot{\gamma}(0)$. Consider the parametrized surface $\sigma$ defined by setting $\left.\sigma\right|_{[0, T] \times s}$ to be the leaf-wise geodesic with the initial conditions $(\gamma(s), w(s))$. Then, in particular, $[(\partial \sigma / \partial s)(t, 0)]$ is independent of the choice of the curve $\eta$ and the family $w$. On the one hand, this vector represents $G(t)[v]$ by the definition of the linearized geodesic flow and, on the other, it is $\Gamma(t)[v]$ due to the above description of the holonomy.

To complete the argument, it would be sufficient to show that $\left.G\right|_{T \tilde{\mathscr{F}} \oplus T B}=\Xi$, where we identified $x^{*}(T \tilde{\mathscr{F}} \oplus T B)$ and $\gamma^{*}\left(T \mathscr{F}_{F} \oplus T^{\perp} M\right)$, but this is not true. Let us fix a basis $\xi(0) \in T_{\gamma(0)} \mathscr{F}$. Then, since the metric is flat, $G(t) \xi(0)$ is the basis $\xi(t)$ in $T_{\gamma(t)} \mathscr{F}$ obtained from $\xi(0)$ by the parallel transport along $\gamma$. Let

$$
\xi^{*}(0) \in T_{\gamma(0)}^{*} \mathscr{F}=T_{\gamma(0)}^{\perp} M
$$

be the basis dual to $\xi(0)$. Then $G(t) \xi^{*}(0)=t \xi(t)+\xi^{*}(t) \in T_{\gamma(t)} \mathscr{F}_{F} \oplus T_{\gamma(t)}^{*} \mathscr{F}_{\mathcal{F}}$ in obvious notation. We conclude that $\left.G(t)\right|_{T \tilde{\mathscr{F}} \oplus T B}=\Xi(t)+A(t)$, where

$$
A(t): T_{\gamma(t)}^{*} \mathscr{F} \rightarrow T_{\gamma(t)} \mathscr{F} .
$$

To finish the proof, we argue as when showing in Section 1.2 that the coisotropic Maslov index is independent of the splitting (1-1). With a trivialization fixed, we can view $G$ and $\Phi=\Xi \oplus \Gamma$ as paths in $\operatorname{Sp}(2 n)$. Then, $G$ is homotopic with fixed end-points to the concatenation of $\Phi$ and the path $\Psi(t)=I+A(t)$. All eigenvalues of $\Psi(t)$ are equal to one and therefore $\Delta(\Psi)=0$. Thus, by the additivity and homotopy invariance of the mean index (see, e.g., [Ginzburg and Gürel 2009; Long 2002; Salamon and Zehnder 1992]), we have $\Delta(G)=\Delta(\Phi)=:-\mu(\gamma)$.

Remark 3.2. Proposition 3.1 has the following hypothetical generalization. Assume that $M$ admits a metric with respect to which $\mathscr{F}$ is totally geodesic. Referring the reader to [Usher 2009, Section 7] for a detailed discussion of this condition, we only mention here that it is satisfied when $M$ is Lagrangian (for any metric on $M$ ) and when $M$ is stable. In the latter case, $\mathscr{F}$ is totally geodesic with respect to $\rho$. Then, conjecturally, the mean Conley-Zehnder index of $x$ is equal, up to a sign, to the sum of the mean Morse index of $\gamma$ and $\mu(\gamma)$. When $M$ is stable, the mean Morse index is zero since $\rho$ is flat, and this conjecture reduces to Proposition 3.1. When $M$ is Lagrangian and $x$ is nondegenerate, the conjecture essentially reduces to a well known relation between the Conley-Zehnder, Morse, and Maslov indices. The latter is proved in [Viterbo 1990] using the results from [Duistermaat 1976] in 
the context of the finite-dimensional reduction. A proof relying on the Floer theory version of the Conley-Zehnder index can be found in, for example, [Weber 2002]; see also [Kerman and Şirikçi 2010] for a simple argument.

The next proposition is a substitute for the relation between the Conley-Zehnder and Maslov indices.

Proposition 3.3. Let $K$ be a small perturbation of $\rho$ and $\tilde{x}$ be a nondegenerate periodic orbit of $K$ close to a nontrivial, contractible periodic orbit $x$ of $\rho$. Then

$$
\Delta_{\rho}(x)-n \leq \mu_{\mathrm{CZ}}(\tilde{x}) \leq \Delta_{\rho}(x)+(n-k)
$$

Proof. Note that by the continuity of $\Delta$ and (2-3) we automatically have

$$
\Delta_{\rho}(x)-n \leq \mu_{\mathrm{CZ}}(\tilde{x}) \leq \Delta_{\rho}(x)+n,
$$

regardless of the nature of the flow of $\rho$. Hence only the second inequality in (3-4) requires a proof.

By arguing as in the proof of Proposition 3.1, it is not hard to reduce the proposition to the following linear algebra result. Namely, consider a finite-dimensional symplectic vector space $V$ split as a symplectic direct sum

$$
V=\left(L \oplus L^{*}\right) \oplus E,
$$

where $E$ and $\left(L \oplus L^{*}\right)$ are symplectic spaces, and $L$ and $L^{*}$ are Lagrangian in $L \oplus L^{*}$; see (1-1) and (3-2). Set $\operatorname{dim} V=2 n$ and $\operatorname{dim} L=k$. Consider a path $G:[0,1] \rightarrow \operatorname{Sp}(V)$ of the form $G=A \oplus \Gamma$, where $\Gamma$ is a path in $\operatorname{Sp}(E)$ beginning at $I$ and $A$ is the block-diagonal path

$$
A=\left[\begin{array}{cc}
I & t I \\
0 & I
\end{array}\right]
$$

in $\operatorname{Sp}\left(L \oplus L^{*}\right)$.

Lemma 3.4. Let $\tilde{G}:[0,1] \rightarrow \mathrm{Sp}(V)$ be a small nondegenerate perturbation of $G$, also beginning at $I$. Then

$$
\Delta(G)-n \leq \mu_{\mathrm{CZ}}(\tilde{G}) \leq \Delta(G)+(n-k) .
$$

Proof of the lemma. Again, by (2-2), we have

$$
\Delta(G)-n \leq \mu_{\mathrm{CZ}}(\tilde{G}) \leq \Delta(G)+n,
$$

for any path $G$. Hence, only the second inequality in (3-5) requires a proof.

Next observe that, once the end-point $\Gamma(1)$ is fixed, the path $\Gamma$ is immaterial for the assertion of the lemma. In other words, if the lemma holds for one path with a given end-point, it also holds for every path with the same end-point. This follows from the facts that a homotopy of $G$ can be traced by a homotopy of $\tilde{G}$ 
(both with fixed end-points) and that $\mu_{\mathrm{CZ}}$ and $\Delta$ are invariant under such homotopy and change in the same way when a loop is attached to a path.

As the first step of the proof, let us assume that all eigenvalues of $\Gamma(1)$ are equal to one. Then $\Gamma(1)$ is in the image of the exponential mapping exp for $\operatorname{Sp}(E)$. Indeed, $\Gamma(1)$ is conjugate to a symplectic linear map which can be chosen to be arbitrarily close to $I$; see, for example, [Ginzburg 2010, Lemma 5.5]. Since exp is onto a neighborhood of the identity and commutes with conjugation, $\Gamma(1)$ is in the image of exp. Since 0 is a regular point of exp and the set of regular points is open, we can write $\Gamma(1)=\exp (Q)$, where $Q$ is a regular point of exp and all eigenvalues of $Q$ are equal to zero.

Here we identify the Lie algebra of the symplectic group with the space of quadratic Hamiltonians. As is customary in symplectic geometry, the eigenvalues of $Q$ are, by definition, the eigenvalues of the linear Hamiltonian vector field $X_{Q}$ generated by $Q$. Also note that if we identified $\operatorname{Sp}(E)$ with $\operatorname{Sp}(2(n-k))$ and used the matrix exponential map, we would write $X_{Q}=J Q$ and $\Gamma(1)=\exp (J Q)$.

We have $A(1)=\exp (\rho)$ in $\operatorname{Sp}\left(L \oplus L^{*}\right)$, where $\rho$ is a positive definite form on $L^{*}$ and zero on $L$. Arguing as above, it is not hard to show that $\rho$ is a regular point of exp for $\operatorname{Sp}\left(L \oplus L^{*}\right)$ and that, moreover, $\rho+Q$ is a regular point of the exponential mapping for $\operatorname{Sp}(V)$. Now we have $\tilde{G}(1)=\exp (K)$ in $\operatorname{Sp}(V)$, where the quadratic form $K$ is close to $\rho+Q$. In particular, $K$ is also positive definite on $L^{*}$ and all eigenvalues of $K$ are close to those of $\rho+Q$, that is, close to zero. As has been pointed out above, we can set $\tilde{G}(t)=\exp (t K)$ and $\Gamma(t)=\exp (t Q)$. As a consequence, with our conventions,

$$
\mu_{\mathrm{CZ}}(\tilde{G})=-\operatorname{sgn}(K) / 2 \leq n-k,
$$

where $\operatorname{sgn}(K)$ stands for the signature of $K$ (i.e., the number of positive eigenvalues minus the number of negative eigenvalues); see [Salamon 1999, Section 2.4]. In addition, $\Delta(G)=0$, and we obtain the second inequality of (3-5) in this case. To summarize, we have proved (3-5) when all eigenvalues of $\Gamma(1)$ are equal to one.

To treat the general case, consider the symplectic direct sum decomposition $E=E_{0} \oplus E_{1}$, where $E_{0}$ is spanned by the generalized eigenvectors of $\Gamma(1)$ with eigenvalue one and $E_{1}$ is the symplectic orthogonal complement of $E_{0}$ in $E$. Clearly, $\Gamma(1)$ preserves this decomposition and, after altering if necessary the path $\Gamma$, we may assume that so do all maps $\Gamma(t)$. When $\tilde{G}(1)$ is sufficiently close to $G(1)$, we have the decomposition $V=V_{0} \oplus V_{1}$ preserved by $\tilde{G}(1)$, where $V_{0}$ is close to $\left(L \oplus L^{*}\right) \oplus E_{0}$ and $V_{1}$ is close to $E_{1}$. Applying a time-dependent, close to the identity conjugation to $\tilde{G}(t)$, we reduce the problem to the case where $V_{0}=\left(L \oplus L^{*}\right) \oplus E_{0}$ and $V_{1}=E_{1}$. Consider now the paths $G$ and $\tilde{G}$. Both paths begin and end in $\operatorname{Sp}\left(V_{0}\right) \times \operatorname{Sp}\left(V_{1}\right)$, the first path is contained in this subgroup, and the path $\tilde{G}$ is close to $G$. In particular, $\tilde{G}$ is in a tubular neighborhood of the subgroup. 
Projecting $\tilde{G}$ to $\operatorname{Sp}\left(V_{0}\right) \times \operatorname{Sp}\left(V_{1}\right)$, we can further reduce the question to the case where $\tilde{G}$ is a path in $\operatorname{Sp}\left(V_{0}\right) \times \operatorname{Sp}\left(V_{1}\right)$, just as $G$ is. Denote by $G=\left(G_{0}, G_{1}\right)$ and $\tilde{G}=\left(\tilde{G}_{0}, \tilde{G}_{1}\right)$ the corresponding decompositions of the paths. The $E_{0}$-component of $G_{0}(1)$ is the map $\left.\Gamma(1)\right|_{E_{0}}$ with all eigenvalues equal to one, and hence (3-5) has already been proved for $G_{0}$ :

$$
\Delta\left(G_{0}\right)-\operatorname{dim} V_{0} / 2 \leq \mu_{\mathrm{CZ}}\left(\tilde{G}_{0}\right) \leq \Delta\left(G_{0}\right)+\left(\operatorname{dim} V_{0} / 2-k\right) .
$$

On the other hand, the path $\tilde{G}_{1}$ is a small perturbation of the path $\left.\Gamma\right|_{E_{1}}$. Thus, we have

$$
\Delta\left(G_{1}\right)-\operatorname{dim} V_{1} / 2 \leq \mu_{\mathrm{CZ}}\left(\tilde{G}_{1}\right) \leq \Delta\left(G_{1}\right)+\operatorname{dim} V_{1} / 2 .
$$

Recall that $\Delta(G)=\Delta\left(G_{0}\right)+\Delta\left(G_{1}\right)$ and $\mu_{\mathrm{CZ}}(\tilde{G})=\mu_{\mathrm{CZ}}\left(\tilde{G}_{0}\right)+\mu_{\mathrm{CZ}}\left(\tilde{G}_{1}\right)$ and that $\operatorname{dim} V_{0}+\operatorname{dim} V_{1}=\operatorname{dim} V=2 n$. Thus, adding up these inequalities, we obtain (3-5), which completes the proof of the lemma and hence the proof of the proposition.

3.2. Action selector for "pinned" Hamiltonians, following E. Kerman. Our goal in this section is to describe a construction of an action selector for "pinned" Hamiltonians, which was introduced in [Kerman 2009; Kerman and Şirikçi 2010]. Although the class of Hamiltonians and manifolds we work with is somewhat different from those in the references just given, the action selector is essentially the same as the one considered there. As far as the proofs are concerned, we adopt here the line of reasoning from [Ginzburg 2007] rather than following the Hofergeometric approach from [Kerman 2009]. Since the arguments are quite standard, for the sake of brevity, we just outline the proofs.

Let $M^{2 n-k}$ be a closed submanifold, not necessarily coisotropic, of a symplectic manifold $W^{2 n}$. As before, we require $W$ to be symplectically aspherical and either closed or a geometrically bounded and wide. We assume that $M$ is displaceable and fix a displaceable open set $U$ containing $M$. Denote by $\mathscr{H}$ the collection of nonnegative, autonomous Hamiltonians $H: W \rightarrow \mathbb{R}$ supported in $U$, constant on a small tubular neighborhood of $M$ and attaining the absolute maximum $C:=\max H$, depending on $H$, on this neighborhood. Let us require furthermore that $C>e(U)$, where $e(U)$ is the displacement energy of $U$.

It is easy to see that $\operatorname{HF}_{n}^{(C-\delta, C+\delta)}(H)=\mathbb{Z}_{2}$ once $H \in \mathscr{H}$ and $\delta>0$ is sufficiently small. In fact, $\mathrm{HF}_{*}^{(C-\delta, C+\delta)}(H)=\mathrm{H}_{*+n-k}\left(M ; \mathbb{Z}_{2}\right)$. Furthermore, when $a>C$ is large enough (namely, if $a>C+e(U)$ ), the inclusion map

$$
i_{a}: \mathbb{Z}_{2} \cong \mathrm{HF}_{n}^{(C-\delta, C+\delta)}(H) \rightarrow \operatorname{HF}_{n}^{(C-\delta, a)}(H)
$$

is zero. The proof of this fact is, for example, contained in the proof of [Ginzburg 2007, Proposition 4.1]; see also [Kerman 2009] for the case of closed manifolds. This is the main point of the argument where we need to assume that $W$ is wide 
[Gürel 2008], unless $W$ is closed. For $H \in \mathscr{H}$, set

$$
\mathrm{c}(H)=\inf \left\{a>C \mid i_{a}=0\right\} .
$$

(Strictly speaking, here we have to require $a>C+\delta$ and then also take infimum over all sufficiently small $\delta>0$.) This is a version of the action selector for "pinned" Hamiltonians, introduced in [Kerman 2009].

Alternatively and more explicitly, the action selector c can be defined as follows. Let $\tilde{H}$ be a $C^{2}$-small, nondegenerate perturbation of $H$, also supported in $U$ (or, to be more precise, in $S^{1} \times U$ ) and such that $\tilde{H} \geq H$. Let us also assume that $\tilde{H}$ is autonomous on a small neighborhood of $M$ and that $\max \tilde{H}=C=\max H$ is attained at $p \in M$. (In what follows, we will have $p$ fixed and independent of $\tilde{H}$.) Then $p$, viewed as an element of degree $n$ in the Floer complex $\mathrm{CF}_{*}^{(C-\delta, \infty)}(\tilde{H})$, is exact and there exists a chain in $\mathrm{CF}_{n+1}^{(C-\delta, \infty)}(\tilde{H})$ mapped to $p$ by the Floer differential; see the proof of [Ginzburg 2007, Proposition 4.1]. Let us consider all such chains and, within every chain, pick an orbit with the largest action and then among the resulting orbits we choose an orbit $\tilde{x}$ with the least action. In other words, to obtain $\tilde{x}$, we first maximize the action within every chain and then minimize the result among all chains which are primitives of $p$. Clearly, the orbit $\tilde{x}$ is in general not unique, but the action $\mathscr{A}_{\tilde{H}}(\tilde{x})$ is defined unambiguously.

Let us now set $\mathrm{c}(\tilde{H})=\mathscr{A}_{\tilde{H}}(\tilde{x})$. Then $\mathrm{c}(H)$ is the infimum or the limit (in the obvious sense) of $\mathrm{c}(\tilde{H})$ over all such perturbations $\tilde{H}$ of $H$. (It is clear that $\mathrm{c}(H)$ is less than or equal to the limit; the fact that $\mathrm{c}(H)$ is greater than or equal to the limit is a consequence of the definition of the Floer homology for degenerate Hamiltonians such as $H$.)

It follows from this description that there exists an orbit $x$ of $H$, referred to in what follows as a special one-periodic orbit of $H$, obtained as a limit point of the orbits $\tilde{x}$ in the space of loops as $\tilde{H} \rightarrow H$, such that

$$
C<\mathscr{A}_{H}(x)=\mathrm{c}(H)<C+e(U) \quad \text { and } \quad 1 \leq \Delta(x) \leq 2 n+1 .
$$

Here the upper bound on the action is established by a variant of the standard argument relating action change and the displacement energy; see, e.g., [Ginzburg 2005; Gürel 2008; Hofer and Zehnder 1994; Kerman 2009] and references therein. The lower bound on action is clear for $\tilde{H}$ and $\tilde{x}$. By continuity of the action (with a little extra argument showing that the inequalities are strict) it also holds for $H$ and $x$. The bounds for the index follow from the continuity of the mean index and (2-3). Note that, in general, the special orbit $x$ is not unique.

Remark 3.5. There appears to be no reason to expect the orbit $\tilde{x}$ to be necessarily connected to $p$ by a Floer downward trajectory. However, there exists an orbit $\hat{x}$ of $\tilde{H}$ with this property and such that $C<\mathscr{A}_{\tilde{H}}(\hat{x}) \leq \mathscr{A}_{\tilde{H}}(\tilde{x})$. This is an immediate 
consequence of the definition of $\tilde{x}$. Carefully passing to the limit as $\tilde{H} \rightarrow H$ we obtain an orbit $x^{\prime}$ of $H$ such that $C<\mathscr{A}_{H}\left(x^{\prime}\right) \leq \mathscr{A}_{H}(x)$ and $x^{\prime}$ is connected to $M$ by a Floer downward trajectory. See [Ginzburg 2007] and, in particular, the proofs of Propositions 4.1 and 5.1 therein for the proofs of these facts; note also that $\hat{x}$ is denoted by $\gamma$ in [Ginzburg 2007, Proposition 4.1]. The existence of the orbit $x^{\prime}$ is essential for showing that, in (1-3), the area bounded by $\eta$ is strictly positive.

We refer the reader to [Kerman 2009] for a detailed investigation of the properties of the action selector $\mathrm{c}$. One of these is particularly important for our argument.

Proposition 3.6 [Kerman 2009]. The action selector c is Lipschitz, with Lipschitz constant equal to one, on $\mathscr{H}$ equipped with the sup-norm.

As an immediate consequence of the proposition, the selector $\mathrm{c}$ extends from $\mathscr{H}$ to the $C^{0}$-closure of $\mathscr{H}$ in the space of continuous functions supported in $U$ and this extension is again Lipschitz with Lipschitz constant equal to one. For the sake of completeness, we touch upon a proof of the proposition.

Outline of the proof. Let $H$ and $K$ be two Hamiltonians in $\mathscr{H}$. Consider the perturbations $\tilde{H}$ and $\tilde{K}$ as above. Clearly, it suffices to show that

$$
|\mathrm{c}(\tilde{H})-\mathrm{c}(\tilde{K})| \leq\|\tilde{H}-\tilde{K}\|_{\mathrm{H}},
$$

where

$$
\|F\|_{\mathrm{H}}:=\int_{0}^{1}\left(\max _{W} F_{t}-\min _{W} F_{t}\right) d t
$$

stands for the Hofer norm of $F$.

Denote by $\tilde{x}$ again a least action primitive of $p$ in $\mathrm{CF}_{*}^{(C-\delta, \infty)}(\tilde{H})$ described above. In particular, $\mathrm{c}(\tilde{H})=\mathscr{A}_{\tilde{H}}(\tilde{x})$. It is not hard to see that under the linear homotopy from $\tilde{H}$ to $\tilde{K}$, the orbit $\tilde{x}$ is mapped to a primitive $\tilde{y}=\sum \tilde{y}_{i}$ of $p$ in the complex $\mathrm{CF}_{*}^{(C-\delta, \infty)}(\tilde{K})$, but not necessarily to a least action primitive. In any case, $\mathrm{c}(\tilde{K}) \leq \mathscr{A}_{\tilde{K}}(\tilde{y}):=\max \mathscr{A}_{\tilde{K}}\left(\tilde{y}_{i}\right)$. Meanwhile, a standard calculation yields

$$
\mathscr{A}_{\tilde{K}}(\tilde{y})-\mathscr{A}_{\tilde{H}}(\tilde{x}) \leq\|\tilde{H}-\tilde{K}\|_{\mathrm{H}} .
$$

Hence, we also have $\mathrm{c}(\tilde{K})-\mathrm{c}(\tilde{H}) \leq\|\tilde{H}-\tilde{K}\|_{\mathrm{H}}$. A similar argument, but using the homotopy from $\tilde{K}$ to $\tilde{H}$, shows that $\mathrm{c}(\tilde{H})-\mathrm{c}(\tilde{K}) \leq\|\tilde{H}-\tilde{K}\|_{\mathrm{H}}$, and (3-7) follows.

Remark 3.7. It is worth pointing out that the main advantage of using the action selector for pinned Hamiltonians in the proof of the main theorem over the ordinary action selector is that the former enables us to determine the location of the special orbit $x$ via Lemma 3.8 without additional requirements on $M$ such as that $M$ has restricted contact type. This results in sharper index and energy bounds that we would have otherwise; see [Ginzburg 2007]. 
3.3. Proof of Theorem 1.5. Throughout the proof, as in Section 2.1, a neighborhood of $M$ in $W$ is identified with a neighborhood of $M$ in $M \times \mathbb{R}^{k}$ equipped with the symplectic form $\omega=\omega_{M}+\sum_{j=1}^{k} d\left(p_{j} \alpha_{j}\right)$. Using this identification, we denote by $U_{R}$ or just $U$, with $R>0$ sufficiently small, the neighborhood of $M$ in $W$ corresponding to $M \times B_{R}^{k}$. (Thus, $U_{R}=\left\{\rho<R^{2} / 2\right\}$.) Also set $|p|:=\sqrt{2 \rho}$.

The proof of the theorem relies on a method, by now quite standard, developed in [Viterbo 1990]. The first, albeit technical, step is to specify the class of "test" Hamiltonians.

3.3.1. The Hamiltonians. Fix two real constants $r>0$ and $\epsilon>0$ with $\epsilon<r<R$ and a constant $C>e(U)$. Let $H:[0, R] \rightarrow \mathbb{R}$ be a smooth, nonnegative, (nonstrictly) decreasing function such that

- on $[0, \epsilon]$ the function $H$ is a positive constant $C$,

- on $[\epsilon, 2 \epsilon]$ the function $H$ is concave (i.e., $H^{\prime \prime} \leq 0$ ),

- on $[2 \epsilon, r-\epsilon]$ the function $H$ is linear decreasing from $C-\epsilon$ to $\epsilon$,

- on $[r-\epsilon, r]$ the function $H$ is convex (i.e., $H^{\prime \prime} \geq 0$ ),

- on $[r, R]$ the function $H$ is identically zero.

Abusing notation, we also denote by $H$ the function equal to $H(|p|)$ on $U$ and equal to zero outside $U$. Let us fix the value of the parameter $r$, which is not essential for what follows. The parameters $C$ and $\epsilon$ will vary and we consider the family of functions $H=H_{C, \epsilon}$ parametrized by $C$ and $\epsilon$ and depending smoothly on these parameters.

Clearly, $H \in \mathscr{H}$ for any choice of $\epsilon$ and $C$. As $\epsilon \rightarrow 0$, the functions $H_{C, \epsilon}$ converge uniformly to the continuous functions $H_{C, 0}$ equal to $C$ on $M$, zero outside $U_{r}$, and depending linearly of $|p|$ on $U_{r}$. It is clear that the limit functions $H_{C, 0}$ are continuous in $C$. Thus, by Proposition 3.6, $\mathrm{c}\left(H_{C, \epsilon}\right)$ is a continuous function of $C$ and $\epsilon$ including the limit value $\epsilon=0$. Moreover, the function $C \mapsto \mathrm{c}\left(H_{C, 0}\right)$ is Lipschitz with Lipschitz constant equal to one.

Denote by $X$ the Hamiltonian vector field of the function $|p|$ on $U \backslash M$. By Proposition 2.3, the integral curves of $X$ project to the geodesics of the leaf-wise metric $\rho$ on $M$, parametrized by arc length. The Hamiltonian vector field of $H$ is

$$
X_{H}=H^{\prime} X \text {, }
$$

where $H^{\prime}$ stands for the derivative of $H$ with respect to $|p|$. Note that even though $X$ is defined only on $U \backslash M$, the vector field $X_{H}$ is defined everywhere, for $H$ is constant near $M$ and outside $U_{r}$. Thus, nontrivial one-periodic orbits of $X_{H}$ lie on the levels $|p|=$ const with $H^{\prime}(|p|)$ in the length spectrum $\mathscr{Y}$ of the metric $\rho$. (Recall that, by definition, $\mathscr{S}$ is formed by the lengths of nontrivial closed leafwise geodesics of $\rho$. Here, we may restrict our attention only to the geodesics 
contractible in $W$.) Observe that the "coordinates" $p_{i}$ are constant along the orbits of the flow of $X_{H}$. In other words, every trajectory starting in $U$ lies on a coisotropic submanifold $M \times p \subset U$. This is a particular case of conservation of momentum.

Let $x$ be a nontrivial one-periodic orbit of $H$. A direct calculation relying on Proposition 2.2 shows that

$$
\begin{aligned}
\mathscr{A}_{H}(x) & =H(x)+\mathscr{A}(x) \\
& =H(x)+\mathscr{A}(\pi(x))-|p(x)| l(\pi(x)),
\end{aligned}
$$

where $l$ and $\mathscr{A}$ stand for the length of the curve and, respectively, the negative symplectic area bounded by the curve.

Assume that the slope of $H$ (on the interval $[2 \epsilon, r-\epsilon]$ ) is outside $\mathscr{Y}$. (This is a generic condition.) Then the orbit $x$ lies on the level where $|p(x)|$ is either in the range $[\epsilon, 2 \epsilon]$ or in the range $[r-\epsilon, r]$. Let now $x$ be a special one-periodic orbit from Section 3.2 such that, in particular, (3-6) holds. The key to the proof is the following lemma, which specifies the location of $x$ for, at least, some sequence of the Hamiltonians $H$.

Lemma 3.8. There exists a sequence $C_{j} \rightarrow \infty$ such that the slopes of all functions $H_{C_{j}, \epsilon}$, with $\epsilon>0$ sufficiently small, are outside $\mathscr{Y}$ and $|p(x)| \in[\epsilon, 2 \epsilon]$.

In the Lagrangian case this observation can be traced back to the original work of Viterbo [1990]. Here we follow the treatment from [Kerman 2009] with several modifications resulting from our somewhat different conventions and more importantly from the fact that $M$ is now coisotropic.

Proof of Lemma 3.8. The slope of the function $H_{C, 0}$ is $C / r$. This slope is in $\mathscr{S}$ if and only if $C \in r \mathscr{Y}$ in the obvious notation. The set $\mathscr{Y}$ (and hence $r \mathscr{Y}$ ) is closed, and the slope of $H_{C, \epsilon}$ is close to the slope of $H_{C, 0}$ when $\epsilon>0$ is small. As a consequence, the slope of $H_{C, \epsilon}$ is outside $\mathscr{S}$ whenever $C \notin r \mathscr{S}$ and $\epsilon>0$ is small.

Pick $C \notin r \mathscr{S}$ and a positive sequence $\epsilon_{i} \rightarrow 0$. Without loss of generality, we may require all $\epsilon_{i}$ to be sufficiently close to zero to ensure that the slope of $H_{i}:=H_{C, \epsilon_{i}}$ is not in $\mathscr{Y}$. Let $x_{i}$ be a special orbit of $H_{i}$. Since the norms of the differentials $d H_{i}$ are bounded from above, the norms of the derivatives $\dot{x}_{i}$ are point-wise bounded. By the Arzela-Ascoli theorem, we may assume, after passing if necessary to a subsequence, that the orbits $x_{i}$ converge to a curve $y$ lying on a level $|p|=$ const including possibly the submanifold $M$. It is clear that $y$ is smooth and projects to a closed, leaf-wise geodesic on $M$. Furthermore,

$$
\mathscr{A}_{H_{i}}\left(x_{i}\right)=\mathrm{c}\left(H_{i}\right) \rightarrow H_{C, 0}(y)+\mathscr{A}(y)=\mathrm{c}\left(H_{C, 0}\right),
$$

by the continuity of the action functional and of the action selector $\mathrm{c}$. 
If $\left|p\left(x_{i}\right)\right|$ is in the range $\left[r-\epsilon_{i}, r\right]$ for all $i$, the orbit $y$ is on the level $|p|=r$ and $H_{C, 0}(y)=0$. Thus, we then have

$$
\mathrm{c}\left(H_{C, 0}\right)=\mathscr{A}(y) \in \Sigma,
$$

where $\Sigma$ is the action spectrum or, to be more precise, the symplectic area spectrum of the level $|p|=r$, that is, the collection of symplectic areas bounded by contractible closed characteristics on this level.

Arguing by contradiction, assume now that the lemma fails, that is, for every sufficiently large $C$, say $C>a$, which is not in $r \mathscr{Y}$, there exists such a sequence $\epsilon_{i}$ with $\left|p\left(x_{i}\right)\right|$ in the range $\left[r-\epsilon_{i}, r\right]$. Consider the function $f(C):=\mathrm{c}\left(H_{C, 0}\right)$ on the interval $[a, \infty)$. By (3-8), $f$ sends the set $[a, \infty) \backslash r \mathscr{Y}$ to $\Sigma$. Recall that $r \mathscr{Y}$ is not only closed, but also has zero measure; see [Ginzburg 2007, Lemma 6.6]. By Proposition 3.6, $f$ is a Lipschitz function and, as is well known [Hofer and Zehnder 1994], $\Sigma$ has measure zero. To summarize, $f$ is a Lipschitz function sending a full measure set to a zero measure set. Such a function is necessarily constant. This is impossible, for $f(C) \geq C$ by (3-6).

Let us fix one of the constants $C=C_{j}$ from Lemma 3.8 and let $H_{i}=H_{C_{j}, \epsilon_{i}}$. Denote by $x_{i}$, or just $x$, its one-periodic orbit such as in the lemma. (For the proof of the theorem we do not need the entire double sequence, but only one family of Hamiltonians $H_{C_{j}, \epsilon_{i}}$ parametrized by $\epsilon_{i}$.) Clearly, $\gamma_{i}=\pi\left(x_{i}\right)$ is a leaf-wise geodesic on $M$. Since the slopes of Hamiltonians $H_{i}$ are bounded from above (by, say, $2 C_{j} / r$ ), it is easy to prove using the Arzela-Ascoli theorem that the geodesics $\gamma_{i}$ converge as $i \rightarrow \infty$ after if necessary passing to a subsequence. Denote the limit geodesic (traversed in the opposite direction) by $\eta$. Our goal is to show that $\eta$ has the required properties (1-2) and (1-3). The fact that, by Lemma 3.8, $\left|p\left(x_{i}\right)\right| \in\left[\epsilon_{i}, 2 \epsilon_{i}\right]$ (i.e., $x_{i}$ lies in the region where $H_{i}$ is concave) will be essential for proving this.

3.3.2. Index bounds. Consider a perturbation $\tilde{H}$ of $H=H_{i}$ as in Section 3.2. This Hamiltonian has a one-periodic orbit $\tilde{x}$, a perturbation of $x=x_{i}$, with index $n+1$. After reparametrizing $x$ and reversing its orientation, we can view $x$ as a periodic orbit $x^{-}$of $\rho$. Likewise, $\tilde{x}$ can be viewed as a periodic orbit $\tilde{x}^{-}$of a nondegenerate perturbation $K$ of $\rho$. Denote by $\gamma^{-}=\pi\left(x^{-}\right)$the geodesic $\gamma=\gamma_{i}$ with reversed orientation.

By Proposition 3.1, we have

$$
\mu\left(\gamma^{-}\right)=-\Delta_{\rho}\left(x^{-}\right)
$$

and thus, by Proposition 3.3,

$$
-\mu\left(\gamma^{-}\right)-n \leq \mu_{\mathrm{CZ}}\left(\tilde{x}^{-}\right) \leq-\mu\left(\gamma^{-}\right)+(n-k) .
$$


It is not hard to show that $\mu_{\mathrm{CZ}}\left(\tilde{x}^{-}\right)=-\mu_{\mathrm{CZ}}(\tilde{x})=-(n+1)$ using the fact that $x$ is in the region where $H$ is concave (i.e., $|p(x)| \in\left[\epsilon_{i}, 2 \epsilon_{i}\right]$ ) by Lemma 3.8. As a consequence,

$$
n+1 \leq \mu\left(\gamma^{-}\right)+n \quad \text { and } \quad \mu\left(\gamma^{-}\right)-n+k \leq n+1 .
$$

Hence,

$$
1 \leq \mu\left(\gamma^{-}\right) \leq 2 n+1-k .
$$

Passing to the limit and using the continuity of the mean index, we conclude that the same holds for $\eta$, the limit of the curves $\gamma^{-}$. This proves (1-2).

Remark 3.9. If we had used here just the second inequality of (3-6) rather than Proposition 3.3, we would have the weaker bound $1 \leq \mu\left(\gamma^{-}\right) \leq 2 n+1$.

3.3.3. Action bounds. By the first inequality in (3-6), we have

$$
C<\mathscr{A}_{H}(x)=H(x)+\mathscr{A}(\gamma)-|p(x)| l(\gamma)<C+e(U) .
$$

Here, by the definition of $H$ and Lemma 3.8, $|p(x)| \in\left[\epsilon_{i}, 2 \epsilon_{i}\right]$ and $H(x) \in[C, C-$ $\left.\epsilon_{i}\right]$. Note that the sequence $l(\gamma)$ with $\gamma=\gamma_{i}$ is bounded as $i \rightarrow \infty$ due the fact that the slope of $H_{i}$ is bounded. Thus, passing to the limit (for a subsequence if necessary), we have $0 \leq-\mathscr{A}(\eta) \leq e(U)$. Here, the negative sign comes from the fact that $\eta$ is the limit of $\gamma^{-}$, that is, the geodesics $\gamma$ with reversed orientation. Taking $r>0$ sufficiently small, we obtain

$$
0 \leq \operatorname{Area}(\eta) \leq e(M)+\delta,
$$

for any given $\delta>0$, where $\operatorname{Area}(\eta)=-\mathscr{A}(\eta)$ is the symplectic area bounded by $\eta$. To finish the proof, we need to ensure that the first inequality is strict: $\operatorname{Area}(\eta)>0$. This is an immediate consequence of the non-trivial fact that, by [Ginzburg 2007, Theorem 6.1], $\mathscr{A}_{H}\left(x^{\prime}\right)-C \geq \epsilon$ for some $\epsilon>0$ independent of $i$, where $x^{\prime}$ is the orbit mentioned in Remark 3.5. For then we also have $\mathscr{A}_{H}(x)-C \geq \epsilon$ and, by the first inequality in (3-9), $\operatorname{Area}\left(\gamma^{-}\right)>\epsilon / 2$ when $i$ is large enough. This concludes the proof of (1-3), and thus the proof of the theorem.

Acknowledgments. The author is grateful to Marta Batoreo, Başak Gürel and Ely Kerman for useful discussions and remarks. He also thanks Yael Karshon and Fabian Ziltener for an inspiring discussion of the notion of the coisotropic Maslov index.

\section{References}

[Albers and Frauenfelder 2008] P. Albers and U. Frauenfelder, "Infinitely many leaf-wise intersection points on cotangent bundles", Preprint, 2008. arXiv 0812.4426

[Albers and Frauenfelder 2010] P. Albers and U. Frauenfelder, "Leaf-wise intersections and Rabinowitz Floer homology”, J. Topol. Anal. 2:1 (2010), 77-98. MR 2646990 Zbl 1196.53050 
[Audin et al. 1994] M. Audin, F. Lalonde, and L. Polterovich, "Symplectic rigidity: Lagrangian submanifolds", pp. 271-321 in Holomorphic curves in symplectic geometry, edited by M. Audin and J. Lafontaine, Progr. Math. 117, Birkhäuser, Basel, 1994. MR 1274934

[Banyaga 1980] A. Banyaga, "On fixed points of symplectic maps", Invent. Math. 56 (1980), 215229.

[Bolle 1996] P. Bolle, "Une condition de contact pour les sous-variétés coïsotropes d'une variété symplectique”, C. R. Acad. Sci. Paris Sér. I Math. 322:1 (1996), 83-86. MR 97e:58072

[Bolle 1998] P. Bolle, "A contact condition for $p$-codimensional submanifolds of a symplectic manifold ( $2 \leq p \leq n)$ ", Math. Z. 227:2 (1998), 211-230. MR 99a:58028 Zbl 0894.53035

[Cieliebak et al. 2004] K. Cieliebak, V. L. Ginzburg, and E. Kerman, "Symplectic homology and periodic orbits near symplectic submanifolds", Comment. Math. Helv. 79:3 (2004), 554-581. MR 2005f:53160 Zbl 1073.53118

[Dragnev 2008] D. L. Dragnev, "Symplectic rigidity, symplectic fixed points, and global perturbations of Hamiltonian systems", Comm. Pure Appl. Math. 61:3 (2008), 346-370. MR 2009i:53091 Zbl 1141.37022

[Duistermaat 1976] J. J. Duistermaat, "On the Morse index in variational calculus", Advances in Math. 21:2 (1976), 173-195. MR 58 \#31190 Zbl 0361.49026

[Ekeland and Hofer 1989] I. Ekeland and H. Hofer, "Two symplectic fixed-point theorems with applications to Hamiltonian dynamics", J. Math. Pures Appl. (9) 68:4 (1989), 467-489. MR 91m: 58059 Zbl 0709.58017

[Floer et al. 1994] A. Floer, H. Hofer, and K. Wysocki, "Applications of symplectic homology. I", Math. Z. 217:4 (1994), 577-606. MR 95h:58051 Zbl 0869.58012

[Ginzburg 1999] V. L. Ginzburg, "Hamiltonian dynamical systems without periodic orbits", pp. 3548 in Northern California Symplectic Geometry Seminar, Amer. Math. Soc. Transl. Ser. 2 196, Amer. Math. Soc., Providence, RI, 1999. MR 2001c:37055 Zbl 0958.37044

[Ginzburg 2005] V. L. Ginzburg, "The Weinstein conjecture and theorems of nearby and almost existence", pp. 139-172 in The breadth of symplectic and Poisson geometry, edited by J. E. Marsden and T. S. Ratius, Progr. Math. 232, Birkhäuser, Boston, MA, 2005. MR 2005m:53168

[Ginzburg 2007] V. L. Ginzburg, "Coisotropic intersections", Duke Math. J. 140:1 (2007), 111-163. MR 2009h:53200 Zbl 1129.53062

[Ginzburg 2010] V. L. Ginzburg, “The Conley conjecture”, Ann. of Math. (2) 172:2 (2010), 11271180. MR 2680488 Zbl 05808583

[Ginzburg and Gürel 2003] V. L. Ginzburg and B. Z. Gürel, "A $C^{2}$-smooth counterexample to the Hamiltonian Seifert conjecture in $\mathbb{R}^{4}$ ”, Ann. of Math. (2) 158:3 (2003), 953-976. MR 2005e:37133

[Ginzburg and Gürel 2004] V. L. Ginzburg and B. Z. Gürel, "Relative Hofer-Zehnder capacity and periodic orbits in twisted cotangent bundles", Duke Math. J. 123:1 (2004), 1-47. MR 2005d:53140

[Ginzburg and Gürel 2009] V. L. Ginzburg and B. Z. Gürel, "Periodic orbits of twisted geodesic flows and the Weinstein-Moser theorem", Comment. Math. Helv. 84:4 (2009), 865-907. MR 2010j: 53185

[Gürel 2008] B. Z. Gürel, "Totally non-coisotropic displacement and its applications to Hamiltonian dynamics”, Commun. Contemp. Math. 10:6 (2008), 1103-1128. MR 2009j:53119

[Gürel 2010] B. Z. Gürel, "Leafwise coisotropic intersections”, Int. Math. Res. Not. 2010:5 (2010), 914-931. MR 2595016

[Gürel $\geq 2011]$ B. Z. Gürel, "Fragility of leafwise coisotropic intersections", In preparation. 
[Hofer 1990] H. Hofer, "On the topological properties of symplectic maps", Proc. Roy. Soc. Edinburgh Sect. A 115:1-2 (1990), 25-38. MR 91h:58042 Zbl 0713.58004

[Hofer and Zehnder 1994] H. Hofer and E. Zehnder, Symplectic invariants and Hamiltonian dynamics, Birkhäuser, Basel, 1994. MR 96g:58001 Zbl 0805.58003

[Kang 2009] J. Kang, "Existence of leafwise intersection points in the unrestricted case", Preprint, 2009. arXiv 0910.2369

[Kerman 2008] E. Kerman, “Displacement energy of coisotropic submanifolds and Hofer's geometry”, J. Mod. Dyn. 2:3 (2008), 471-497. MR 2009c:53129 Zbl 1149.53321

[Kerman 2009] E. Kerman, “Action selectors and Maslov class rigidity”, Int. Math. Res. Not. 2009:23 (2009), 4395-4427. MR 2010k:53133 Zbl 1183.53074

[Kerman and Şirikçi 2010] E. Kerman and N. I. Şirikçi, "Maslov class rigidity for Lagrangian submanifolds via Hofer's geometry", Comment. Math. Helv. 85:4 (2010), 907-949. MR 2718142 Zbl 1198.53091

[Long 2002] Y. Long, Index theory for symplectic paths with applications, Progress in Mathematics 207, Birkhäuser, Basel, 2002. MR 2003d:37091 Zbl 1012.37012

[Moser 1978] J. Moser, “A fixed point theorem in symplectic geometry”, Acta Math. 141:1-2 (1978), 17-34. MR 57 \#17714 Zbl 0382.53035

[Oh 2003] Y.-G. Oh, "Geometry of coisotropic submanifolds in symplectic and Kähler manifolds", 2003. arXiv math.SG/0310482

[Polterovich 1991a] L. Polterovich, "Monotone Lagrange submanifolds of linear spaces and the Maslov class in cotangent bundles", Math. Z. 207:2 (1991), 217-222. MR 93c:58075 Zbl 0703. 58018

[Polterovich 1991b] L. V. Polterovich, “The Maslov class of the Lagrange surfaces and Gromov's pseudo-holomorphic curves", Trans. Amer. Math. Soc. 325:1 (1991), 241-248. MR 91h:58046 Zbl 0719.53016

[Salamon 1999] D. A. Salamon, "Lectures on Floer homology", pp. 143-230 in Symplectic geometry and topology (Park City, UT, 1999), edited by Y. Eliashberg and L. Traynor, IAS/Park City Mathematics Series 7, American Mathematical Society, Providence, RI, 1999. MR 2000c:53001

[Salamon and Zehnder 1992] D. Salamon and E. Zehnder, "Morse theory for periodic solutions of Hamiltonian systems and the Maslov index", Comm. Pure Appl. Math. 45:10 (1992), 1303-1360. MR 93g:58028 Zbl 0766.58023

[Sikorav 1994] J.-C. Sikorav, "Some properties of holomorphic curves in almost complex manifolds", pp. 165-189 in Holomorphic curves in symplectic geometry, edited by M. Audin and J. Lafontaine, Progr. Math. 117, Birkhäuser, Basel, 1994. MR 1274929

[Sullivan 1978] D. Sullivan, "A foliation of geodesics is characterized by having no "tangent homologies"”, J. Pure Appl. Algebra 13:1 (1978), 101-104. MR 80c:57019

[Tonnelier 2010] B. Tonnelier, Déplacement des sous-variétés coisotropes; quasi-measures topologiques et symplectiques sur les surfaces, Thèse de doctorat, École Polytechnique, 2010.

[Usher 2009] M. Usher, "Boundary depth in Floer theory and its applications to Hamiltonian dynamics and coisotropic submanifolds", Preprint, 2009. To appear in Israel J. Math. arXiv 0903.0903

[Viterbo 1990] C. Viterbo, "A new obstruction to embedding Lagrangian tori", Invent. Math. 100:2 (1990), 301-320. MR 91d:58085 Zbl 0727.58015

[Weber 2002] J. Weber, "Perturbed closed geodesics are periodic orbits: index and transversality", Math. Z. 241:1 (2002), 45-82. MR 2003j:53137 Zbl 1037.53060 
[Ziltener 2009] F. Ziltener, "A Maslov map for coisotropic submanifolds, leaf-wise fixed points and presymplectic non-embeddings", Preprint, 2009. arXiv 0911.1460

[Ziltener 2010] F. Ziltener, "Coisotropic submanifolds, leaf-wise fixed points, and presymplectic embeddings”, J. Symplectic Geom. 8:1 (2010), 95-118. MR 2609631 Zbl 05794630

Received December 10, 2009. Revised February 26, 2010.

VIKTOR L. GINZBURG

ginzburg@math.ucsc.edu

DEPARTMENT OF MATHEMATICS

UNIVERSITY OF CALIFORNIA

SANTA CRUZ, CA 95064

UNITED STATES

http://math.ucsc.edu/ ginzburg/ 


\title{
PACIFIC JOURNAL OF MATHEMATICS
}

\author{
http://www.pjmath.org \\ Founded in 1951 by
}

E. F. Beckenbach (1906-1982) and F. Wolf (1904-1989)

\section{EDITORS}

V. S. Varadarajan (Managing Editor)

Department of Mathematics

University of California

Los Angeles, CA 90095-1555

pacific@math.ucla.edu

Vyjayanthi Chari

Department of Mathematics

University of California

Riverside, CA 92521-0135

chari@math.ucr.edu

\section{Robert Finn}

Department of Mathematics Stanford University

Stanford, CA 94305-2125

finn@math.stanford.edu

Kefeng Liu

Department of Mathematics

University of California

Los Angeles, CA 90095-1555

liu@math.ucla.edu
Darren Long

Department of Mathematics

University of California

Santa Barbara, CA 93106-3080

long@math.ucsb.edu

Jiang-Hua Lu

Department of Mathematics

The University of Hong Kong

Pokfulam Rd., Hong Kong jhlu@maths.hku.hk

Alexander Merkurjev

Department of Mathematics University of California

Los Angeles, CA 90095-1555 merkurev@math.ucla.edu
Sorin Popa

Department of Mathematics

University of California

Los Angeles, CA 90095-1555

popa@math.ucla.edu

Jie Qing

Department of Mathematics

University of California

Santa Cruz, CA 95064

qing@ cats.ucsc.edu

Jonathan Rogawski

Department of Mathematics

University of California

Los Angeles, CA 90095-1555

jonr@math.ucla.edu

\section{PRODUCTION}

pacific@math.berkeley.edu

Silvio Levy, Scientific Editor Matthew Cargo, Senior Production Editor

ACADEMIA SINICA, TAIPEI

CALIFORNIA INST. OF TECHNOLOGY

INST. DE MATEMÁTICA PURA E APLICADA

KEIO UNIVERSITY

MATH. SCIENCES RESEARCH INSTITUTE

NEW MEXICO STATE UNIV.

OREGON STATE UNIV.

\section{SUPPORTING INSTITUTIONS}

STANFORD UNIVERSITY
UNIV. OF BRITISH COLUMBIA
UNIV. OF CALIFORNIA, BERKELEY
UNIV. OF CALIFORNIA, DAVIS
UNIV. OF CALIFORNIA, LOS ANGELES
UNIV. OF CALIFORNIA, RIVERSIDE
UNIV. OF CALIFORNIA, SAN DIEGO
UNIV. OF CALIF., SANTA BARBARA

UNIV. OF CALIF., SANTA CRUZ

UNIV. OF MONTANA

UNIV. OF OREGON

UNIV. OF SOUTHERN CALIFORNIA

UNIV. OF UTAH

UNIV. OF WASHINGTON

WASHINGTON STATE UNIVERSITY

These supporting institutions contribute to the cost of publication of this Journal, but they are not owners or publishers and have no responsibility for its contents or policies.

See inside back cover or www.pjmath.org for submission instructions.

The subscription price for 2011 is US \$420/year for the electronic version, and \$485/year for print and electronic.

Subscriptions, requests for back issues from the last three years and changes of subscribers address should be sent to Pacific Journal of Mathematics, P.O. Box 4163, Berkeley, CA 94704-0163, U.S.A. Prior back issues are obtainable from Periodicals Service Company, 11 Main Street, Germantown, NY 12526-5635. The Pacific Journal of Mathematics is indexed by Mathematical Reviews, Zentralblatt MATH, PASCAL CNRS Index, Referativnyi Zhurnal, Current Mathematical Publications and the Science Citation Index.

The Pacific Journal of Mathematics (ISSN 0030-8730) at the University of California, c/o Department of Mathematics, 969 Evans Hall, Berkeley, CA 94720-3840, is published monthly except July and August. Periodical rate postage paid at Berkeley, CA 94704, and additional mailing offices. POSTMASTER: send address changes to Pacific Journal of Mathematics, P.O. Box 4163, Berkeley, CA 94704-0163.

PJM peer review and production are managed by EditFLOW ${ }^{\mathrm{TM}}$ from Mathematical Sciences Publishers.

PUBLISHED BY PACIFIC JOURNAL OF MATHEMATICS

at the University of California, Berkeley 94720-3840

A NON-PROFIT CORPORATION

Typeset in IATEX

Copyright $(2011$ by Pacific Journal of Mathematics 


\section{PACIFIC JOURNAL OF MATHEMATICS}

Volume $250 \quad$ No. $1 \quad$ March 2011

Nonconventional ergodic averages and multiple recurrence for von $\quad 1$ Neumann dynamical systems

Tim Austin, TAnja EIsner and Terence TaO

Principal curvatures of fibers and Heegaard surfaces

WILLIAM BRESLIN

Self-improving properties of inequalities of Poincaré type on $s$-John domains

SENG-KeE CHUA and RICHARD L. WHEEDEN

The orbit structure of the Gelfand-Zeitlin group on $n \times n$ matrices

MARK COLARUSSO

On Maslov class rigidity for coisotropic submanifolds

VIKTOR L. GINZBURG

Dirac cohomology of Wallach representations

Jing-Song HuAng, PAVle PANDŽIĆ and Victor PROTSAK An example of a singular metric arising from the blow-up limit in the 191 continuity approach to Kähler-Einstein metrics

YALONG SHI and XIAOHUA ZHU

Detecting when a nonsingular flow is transverse to a foliation

SANDRA SHIELDS

Mixed interior and boundary nodal bubbling solutions for a sinh-Poisson equation

JUNCHENG WeI, LONG WEI and FENG ZHOU 